\title{
Wideband SQUID Amplifiers for Axion Search Experiments
}

\author{
Andrei Matlashov ${ }^{1}$ \\ IBS, Center for Axion and Precision Physics Research, Daejeon, South Korea \\ E-mail: andrei@ibs.re.kr \\ Yannis Semertzidis \\ IBS, Center for Axion and Precision Physics Research, Daejeon, South Korea \\ KAIST, Department of Physics, Daejeon, South Korea \\ E-mail: yannis@kaist.ac.kr
}

\begin{abstract}
The microwave cavity experiment for the dark matter axion search is based on the theoretical prediction that axions could convert into microwave photons inside a High-Q cavity immersed in a strong magnetic field. In such experiments a weak, of order $10^{-22} \mathrm{~W}$, quasi-monochromatic microwave signal should be detected by scanning it in a very wide frequency range. The best cryogenic semiconductor amplifiers have the lowest noise temperature plateau of about $1.0 \mathrm{~K}$ even at significantly lower ambient temperature. Superconducting quantum interference devices, or SQUIDs, can work as microwave amplifiers with temperature noise close to the standard quantum limit that is about $50 \mathrm{mK}$ at $1 \mathrm{GHz}$ [1]. Previously designed SQUID-based high frequency amplifiers have narrow bandwidth due to a microstrip resonant input coil. It requires serial replacements of SQUID preamplifiers in order to scan wide frequency range. This procedure is complex and time consuming because of a large mass of hardware should be cooled down below $100 \mathrm{mK}$. For suitable amplification at high frequency a SQUID should be designed with the smallest possible Josephson junction capacitance, reasonably low SQUID loop inductance, and maximal steepness of the transfer function. Sub-micron size cross-type Josephson junctions have a very low capacitance of about $0.04 \mathrm{pF}$ that is one order smaller than a capacitance of Josephson junctions fabricated using conventional window-type technology. Such junctions initially were designed and used at IPHT for low-frequency SQUID current sensors [2]. Slightly modified current sensors were tested as amplifiers at frequency range $0.5-5 \mathrm{GHz}$. They showed high gain and both wideband and resonant performance. In this paper we describe wideband SQUID microwave amplifiers fabricated at IPHT using sub-micron size Josephson junctions with very low capacitance.
\end{abstract}

The 39th International Conference on High Energy Physics (ICHEP2018)

4-11 July, 2018

Seoul, Korea

\section{${ }^{1}$ Speaker}

(C) Copyright owned by the author(s) under the terms of the Creative Commons 


\section{Introduction}

When a SQUID is biased with proper dc current its output voltage changes periodically with increasing input magnetic flux that is called a volt-flux characteristic or VФ curve. For amplification of a small signal the crucial parameter is its steepness. The main parameters determining the V $\Phi$ curve steepness include SQUID loop inductance, $L_{0}$, Josephson junction critical current, $I_{0}$, its capacitance, $C_{\mathrm{JJ}}$, and a shunt resistor, $R_{\mathrm{SH}}$. The maximal value of the transfer function is given by $\mathrm{d} V / \mathrm{d} \Phi \approx R_{\mathrm{SH}} / 2 L_{0}$ and could be large proportionally to $R_{\mathrm{SH}}$. However, having non-hysteretic VФ curve requires the McCumber-Stewart parameter $\beta_{C}=2 \pi \mathrm{I}_{0} R_{\mathrm{SH}}{ }^{2} C_{\mathrm{JJ}} / \Phi_{0}<1$ that limits the maximum value of $R_{\mathrm{SH}}$. As $L_{0}$ determines the coupling of the input signal to the SQUID, the most elegant way to increase the transfer function is to decrease the Josephson junction capacitance $C_{\mathrm{JJ}}$.

\section{Results}

The SQUID with $30 \mathrm{pH}$ loop inductance and 12-turn input coil was used for tests as a microwave amplifier at temperature of $4.2 \mathrm{~K}$ [3]. Fig. 1 demonstrates the gain vs. frequency plots for two working points positioned on gentle (left plot) and steep (right plot) sides of the VФ curve.
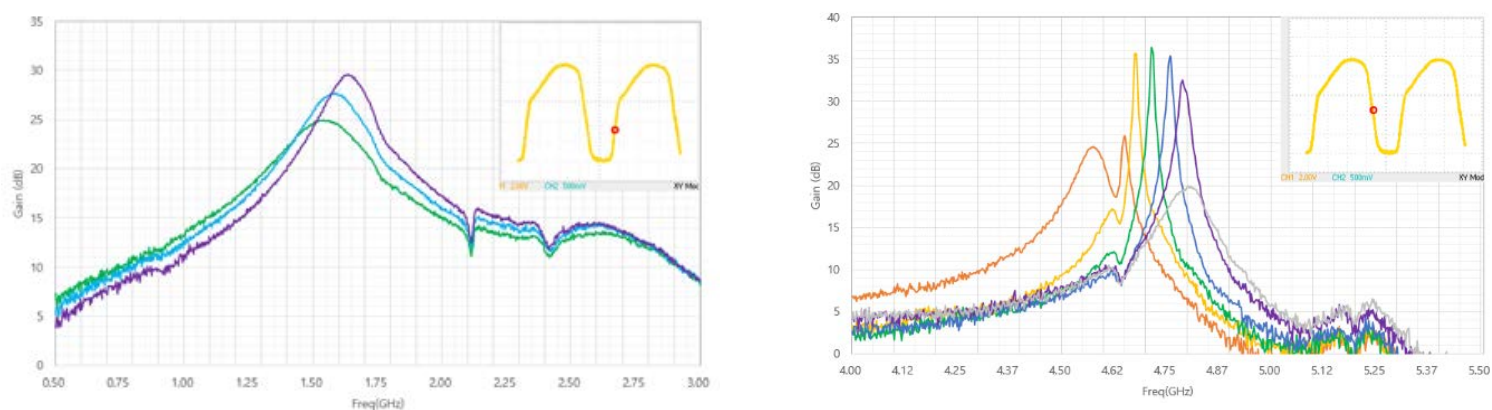

Fig. 1. Power gain vs. frequency plots for the microwave SQUID amplifiers: the corner insertions show the VФ curves with red dot corresponding to the working point positions on the gentle (left plot) and the steep (right plot) sides. The collored plots corresponds to different dc bias currents: 96, 98 and $100 \mu \mathrm{A}$ (left) and from 92 to $100 \mu \mathrm{A}$ with $2 \mu \mathrm{A}$ steps (right).

This amplifier can be set for operation at different frequencies depending on the working point position on the $\mathrm{V} \Phi$ curve. The left plot on figure 1 shows non-resonant wideband amplification exceeding $15 \mathrm{~dB}$ in $1.1-2.3 \mathrm{GHz}$ frequency range and $10 \mathrm{~dB}$ in $0.8-2.9 \mathrm{GHz}$ range, when the working point is located on the gentle side. This amplifier also can be set in a microstrip resonance mode when the working point is located on the steep side. It has more than $30 \mathrm{~dB}$ gain at the resonance frequency depending on the SQUID dc bias current and can be adjusted in the frequency range $4.5-4.8 \mathrm{GHz}$.

\section{References}

[1] M. Mück and R. McDermott, Radio-frequency amplifiers based on dc SQUIDs, Supercond. Sci. Technol. 23, 093001 (2010)

[2] M. Schmelz, et al. A new family of field-stable and highly sensitive SQUID current sensors based on sub-micrometer cross-type Josephson junctions, Supercond. Sci. Technol., 30 (2017) 074010

[3] A.N. Matlashov, M. Schmelz, V. Zakosarenko, R. Stolz and Y. K. Semertzidis, SQUID Amplifiers for Axion Search Experiments", Cryogenics, 91, 125-127 (2018) 